\title{
Development of gases mole fraction and concentration transference procedures with the use of Fourier spectroscopy
}

Leonid Konopelko1a, Vitaly Beloborodov1, Dmitry Rumiantsev1 and Dmitry Selukov1

1D.I.Mendeleyev Metrology Institute (VNIIM), 190005, 19 Moskovsky ave., St. Petersburg, Russia

\begin{abstract}
One of our recent works is development of procedures to transfer mole fraction and mass concentration of $\mathrm{Cl} 2, \mathrm{HCl}, \mathrm{SO} 2, \mathrm{H} 2 \mathrm{~S}, \mathrm{NO}, \mathrm{CO}$ and to improve units transference accuracy from State Primary Measurement Standard of Mole Fraction and Mass Concentration of Components in Gas Media (GET 154-2011 [1]) to working standards of 0-th and 1-st grade (SRM) based on methods of IR spectroscopy.
\end{abstract}

\section{Introduction and goal of this work}

Optical methods of pollution control in different spheres take an important place in a row of analytical measurements. Practically speaking all groups of substances have spectral absorption bands in one or another field of spectrum. This fact makes possible to use as qualitative as well as quantitative spectral analysis to define chemical composition and to measure a content of different gas mixtures. Modern optical instrumentation working in wide spectral regions or in narrow spectral bands and using special computer programs potentially can measure gas concentrations with high precision. But backlog of development of spectral methods from the measurement capabilities of modern instrumentation leads to loose of accuracy and to complication of analyses.

Spectral absorption method of measurement of concentrations of substances is based on comparison of measured spectrum and etalon spectrum which can be received by two ways.

The first way implies receiving of etalon spectrum for concrete spectral instrumentation with the use of standard gas mixtures in cylinders.

The second way of receiving of etalon spectrum is based on spectrum calculation with the use of known spectroscopic parameters of molecules and spectral characteristics of instrumentation. In this case synthetic spectra can be received for great number of gases and vapors of substances in the wide concentration range from 10-9 (for Cavity Ring-
Down Spectroscopy) up till $100 \%$. This way of receiving of etalon spectrum is used e.g. for tasks of metrological assurance of spectral methods as for concentration control of substances in surface layer of atmosphere as well as for content control of substances in atmosphere on large optical paths. At present such way as a rule is used when it is impossible to receive etalon spectrum with the use of standard gas mixtures.

Among available databases used for IR math spectra modeling [2-5] the most widely used at present is the HITRAN database with its updated versions $[4,6,7]$ developed by a group of scientific centers and universities under aegis of the Harvard-Smithsonian Center for Astrophysics Atomic and Molecular Physics Division. The GEISA database [5] is developed by a group of scientific centers and universities under aegis of the Laboratoire de Meteorologie Dynamique, Universite' Pierre et Marie Curie.

Also there are a catalog of cross-sections of large number of substances in the Internet [8] and a database of absorption coefficients of volatile organic compounds made by the NIST [9].

Databases are updated regularly. E.g. updates were made for the first overtone of $12 \mathrm{C} 16 \mathrm{O}$ located near $2.4 \mu \mathrm{m}$. New results show that the line intensities of the HITRAN version [6] were systematically larger by $4.2 \%$ that those in the HITRAN version [7]. Similarly in the case of the second overtone (3-0) of $12 \mathrm{C} 16 \mathrm{O}$ located around $1.6 \mu \mathrm{m}$, measured intensity values were smaller by $5-7 \%$ in the HITRAN version [7] than in the HITRAN version [6]. Furthermore the 
HITRAN includes such parameter like "uncertainty indices" ("lerr"). For CO in the spectral band where were made calculations presented in this paper lerr $=$ 2. For this lerr uncertainty of line position and air induced line shift is between 0.01 and $0.1 \mathrm{~cm}-1$. At the same time line intensity is evaluated like "average or estimate" [7].

At the conferences concerning the spectral databases, e.g. 11th ASA Conference united with the 12th HITRAN Conference, Reims, France, August 29-31, 2012, it was found out that difference between parameters of spectral lines taken from different versions of databases and between GEISA and HITRAN databases can be explained by the fact that for the most part the accuracy of data collected in a database is estimated without any coordinated procedure by scientists who send the data to be included in a database and who work in different laboratories all over the world independently.

Two conditions are needed for reliable measurement of concentration of substance with the use of synthetic spectra:

1. Accurate parameters of spectral lines.

Synthetic spectrum can differ from measured spectrum for a substance with the same concentration. To use calculation methods for measurement, it is needed to introduce correction factor. An example of such work is key comparisons CCQM-P110-B2 (10 ppm NO2 in nitrogen) [10]. Use of the HITRAN database with the correction factor can substantially increase accuracy of measurement of NO2. It is especially important because of possible instability of $\mathrm{NO} 2$ in gas mixture. 2. Precise knowledge of instrument parameters (line shape function of an instrument).

Precise knowledge of line shape function and its stability is needed to use calculation method. It is possible to use a test sealed optical cell containing gas, e.g. $\mathrm{CO}$ or $\mathrm{HBr}$ to find line shape function of an instrument. Gas in a test sealed optical cell should have relatively narrow spectral lines in IR.

Use of such test cell helps to find line shape function of an instrument rather precisely and to control its stability in time introducing needed corrections. It is possible to get once (with the use of standard gas mixtures) a difference between real and calculated concentrations. Later on such kind of measurement can be conducted on another instrument. The only one condition is to use a test sealed optical cell to find line shape function of an instrument.

Accordingly to use available spectroscopic databases successfully in absorption spectroscopic methods it is needed to single out the most precise data and to add respective uncertainty basing on proper experimental investigation conducted with the use of standard gas mixtures. So in this way it will be possible to use spectroscopic databases for measurements without the use of standard gas mixtures. There will be used only a test sealed optical cell to control line shape function.

The goal of this work is theoretical and experimental investigations of:

- Modern FTIR technical and metrological features;

- Spectra of gases of interest;

- Available molecular spectroscopicdatabases;

- Methods of spectral lines handling and methods of instrument line shape control;

- Software and procedures of SRM certification with the help of FTIR using minimal SRM quantity for calibration.

\section{Key comparisons under aegis of the BIPM}

CCQM-P110-B2 key comparison was conducted in 2010 [10]. One of the aims of this comparison was measurement of NO2 concentrations with the use of calculation e.g. using the HITRAN database. VNIIM has taken part in this work with Fourier spectrometer FSM 1201 [10]. NO2 absorption spectrum was taken by analyzing gas mixture in cylinder circulated by the BIPM and calculations were made using NO2 line parameters. Synthetic and experimental NO2 spectra are presented on Fig. 1.

Figure 1. Synthetic and experimental $\mathrm{NO}_{2}$ spectra (blue synthetic spectrum, red - experimental spectrum)

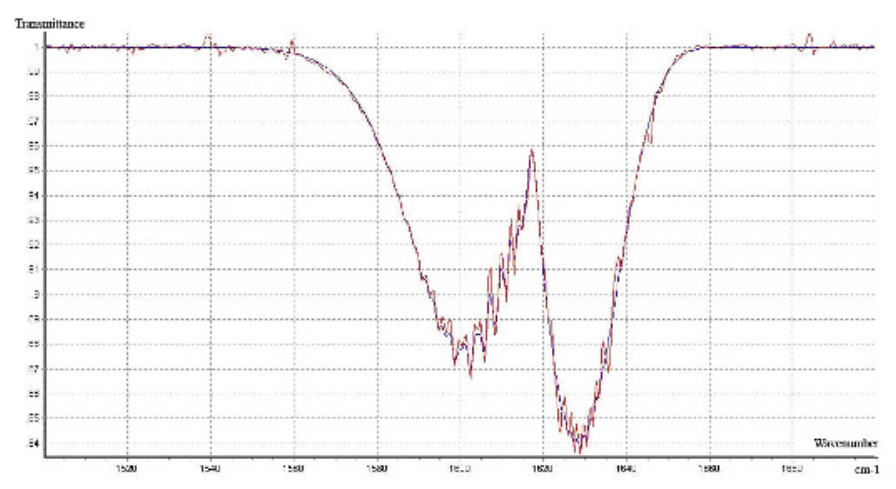

The next results were received: $\mathrm{NO} 2$ concentration in the cylinder under test using calculation method 10.44 ppm; NO2 concentration as reported by the BIPM - $10.32 \mathrm{ppm}$. Difference is about $1.2 \%$. This difference can be explained by uncertainty of the instrument parameters (line shape function) and by uncertainty of line parameters in the HITRAN database. Later on several gas mixtures in cylinders containing 
NO2 were explored in VNIIM (concentration about 500 ppm.). Results received by calculation differed from nominal value in the range from $1.0 \%$ till $1.7 \%$. Thus it is possible to conclude that error of measurement of $\mathrm{NO} 2$ concentration with the use of calculation method can be about $2 \%$.

\section{Algorithm of spectra handling and methods of control of spectral line shape function of FTIR}

To develop an optimal algorithm of control of substances content (Fig. 2.) with the use of calculation method and the HITRAN database an exploration of gas mixtures was conducted. Primary standard gas mixtures were used only once to get correction factors i.e. absorption spectra for these gas mixtures are measured in known temperature $\mathrm{T}$, pressure $\mathrm{P}$ and optical path length $\mathrm{L}$ only once. Then correction factors are calculated using measured absorption spectra and line parameters from the HITRAN database.

One type of standard gas mixture (test sealed optical cell - $\mathrm{CO}$ in nitrogen) is used to control line shape function stability in time. Thus introducing into operation IR spectrophotometers working in accordance with the algorithm presented in Fig. 2 there is no need of SRM to certify analyzed gas mixture.

Software was developed in VNIIM to realize this method of measurement. This software provides calculation of monochromatic absorption spectrum using line parameters from the HITRAN database, provides calculation of convolution of monochromatic absorption spectrum with line shape function of instrument and calculation of substance content in analyzed gas mixture by the least-squares method using synthetic and measured absorption spectra of analyzed substance.
Figure 2. Algorithm of control of substances content.

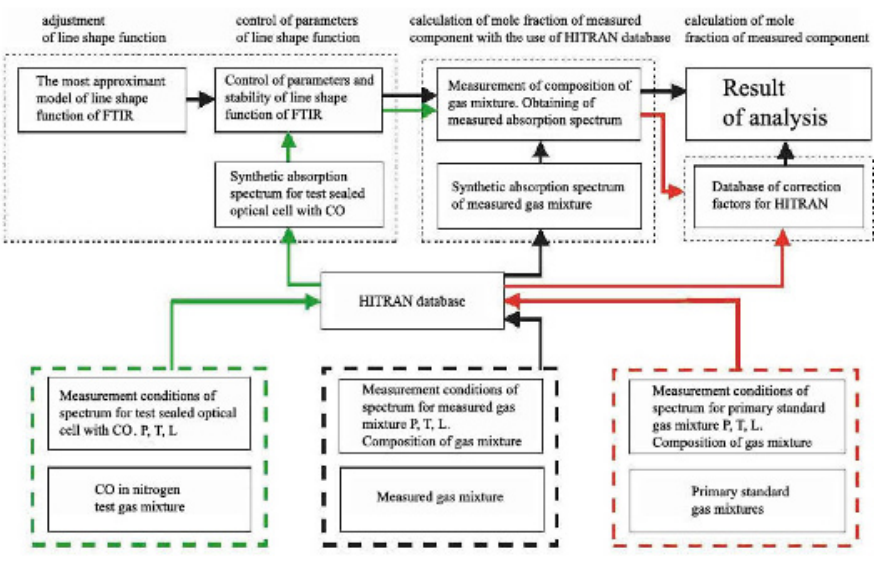

The correction factors which were received in VNIIM as a result of experiments conducted with the use of high-precision gas mixtures are presented in table.

Table. Correction factors.

\begin{tabular}{|c|c|c|}
\hline $\begin{array}{c}\text { Composition of } \\
\text { gas mixture }\end{array}$ & $\begin{array}{c}\text { Range of mole fraction of } \\
\text { measured component in } \\
\text { gas mixture, \% }\end{array}$ & $\begin{array}{c}\text { Correction } \\
\text { factor }\end{array}$ \\
\hline $\mathrm{HCl}+\mathrm{N}_{2}$ & from 0.10 up till 0.30 & 1,054 \\
\hline $\mathrm{CO}+\mathrm{N}_{2}$ & from 0.1 up till 8.0 & 1,050 \\
\hline $\mathrm{NO}+\mathrm{N}_{2}$ & from 0.2 up till 5.0 & 1,056 \\
\hline $\mathrm{SO}_{2}+\mathrm{N}_{2}$ & from 0.05 up till 7.5 & 1,036 \\
\hline
\end{tabular}

It should be emphasized that these correction factors are proved for spectra calculation and calculation of concentration for range of mole fraction presented in table with the use of software developed in VNIIM.

Also it should be mentioned that atmospheric pressure during the experiment was changing $+/-2$ $\mathrm{kPa}$ around normal atmospheric pressure and temperature $+/-2 \mathrm{oC}$ around $22 \mathrm{oC}$.

\section{Conclusion}

The result of this work is development and studying of methods of spectra handling, providing gas concentration of component in analyzed gas mixture. It was substantiated on basis of investigations that optimal algorithm of spectra 
handling is algorithm with the use as a synthetic spectrum which in turn uses special spectroscopic database. Such database includes spectral lines parameters, integral absorption coefficients, adjustment coefficients etc. Also an instrument line shape should be controlled with the use of test SRM (CO in nitrogen).

Adoption of modern FTIR and development of new method of measurement will ensure after it approbation a remote units transfer from State Primary Measurement Standard of Mole Fraction and Mass Concentration of Components in Gas Media GET 154-2011 [1] to manufactured gas mixtures based on methods of IR absorption spectroscopy. This goal is of great practical importance especially for countries with large territory when manufacture of SRM and measurement means are exceedingly geographically distributed.

It might be as well to organize international key comparisons for some other gases in order to make a conclusion on adaptability of calculation methods and its possible uncertainties.

\section{References}

1. L. Konopelko, Y. Kustikov, D. Selukov and V. Beloborodov. 6th international Gas Analysis Symposium (GAS2011), Rotterdam the Netherlands, February 9-11, 2011, CD-ROM 18 pages.

2. Pickett HM, Poynter RL,Cohen EA, Delitsky ML, Pearson JC, Muller HSP. JQSRT; 60, 883-90 (1998)

3. Muller HSP, Schloder F, Stutzki J, Winnewisse rG. J Mol Struct; 742: 215 (2005)

4. L.S. Rothman et al. JQSRT; 110, 533-572 (2009)

5. N. Jacquinet-Husson at al. JQSRT; 112, 2395$2445(2011)$

6. L.S. Rothman et al. JQSRT; 82, 5-44 (2003)

7. L.S. Rothman et al. JQSRT ; 96, 139-204 (2005)

8. Database MPI-Mainz-UV-VIS Spectral Atlas: http://www.atmosphere.mpg.de/enid/2e8b4db4e b1 ebee23ece6ea40b185612,0/Spectra/Introducti on_1rr.html

9. NIST Standard Reference Database 79: http://www.nist.gov/srd/nist79.cfm

10. Edgar Flores et al. Metrologia; 50 (2013), Tech. Suppl., 08011; 97 pages. 